\title{
Discriminability of stimuli in matching to sample
}

\author{
ADRIENNE A. WHYTE and JOHN J. BOREN \\ The American University, Washington, District of Columbia 20016
}

\begin{abstract}
Steady-state accuracy on matching to sample was studied in the pigeon. In Phases 1 and 3 of the experiment, geometric forms were used as stimuli. In Phase 2, the stimuli were colors. In each phase of the experiment, four comparison stimuli were used. Matching accuracy was substantially higher in the color matching phase than in either form matching phase. This result shows that steady-state differences in accuracy exist, under some conditions, between form matching and color matching. This finding extends the theory that differences between form and color matching exist in acquisition.
\end{abstract}

Matching to sample is a conditional discrimination procedure in which a subject is required to select a comparison stimulus which matches a sample stimulus on some preselected dimension. The number and type of stimuli used in matching-to-sample experiments have varied among laboratories. Cumming and Berryman used colors (1961) and geometric forms (1965) as stimuli. They studied the acquisition of matching behavior in the pigeon using a three-key response panel and three colors as stimuli. They found that acquisition of matching under these circumstances followed a predictable sequence. For the first three or four sessions, the subjects were correct on about $50 \%$ (chance) of the trials. Performance rapidly improved until the subjects were matching correctly on approximately $100 \%$ of the trials after 10 to 15 sessions. Cumming and Berryman (1965) also studied matching with geometric forms and found that acquisition of form matching was much slower than acquisition of color matching. Blough (1959) used flickering and steady white lights as stimuli.

Although many types of stimuli have been used in matching-to-sample studies, there have been few studies on the differences in the discriminability of these stimuli. The studies which do exist are concerned with differences in acquisition. A relevant consideration has been the number of sessions needed to reach a $90 \%$ correct criterion. Cumming and Berryman (1965) noted that the acquisition of matching with geometric forms as stimuli is much slower than acquisition of color matching. Mello (1971) found that discriminations in matching between some geometric forms were more difficult to acquire than discriminations between other forms. Farthing and Opuda (1974), in a study of matching

This paper is based on a master's thesis by the first author submitted to The American University in partial fulfillment of the requirements for a master's degree. The research was supported by NIMH Grant MH20785. We would like to acknowledge the guidance and advice of Dr. Alan M. Silberberg. Reprints may be obtained from Adrienne Whyte at Department of Psychology. The American University, Washington, D.C. 20016 . involving both colors (red, blue, and yellow) and forms (triangle, circle, and cross), found that acquisition of color matching to a $90 \%$ correct criterion took approximately 12 sessions while similar acquisition of form matching took about 50 sessions. In all of these studies, the $90 \%$ correct criterion was eventually attained by all subjects. In each case, three-key response panels and no more than two comparison stimuli were used. Cumming and Berryman (1965) suggested that the difficulty of matching increases with increasing numbers of alternative stimuli. In the present study, the subjects were required to discriminate among four comparison stimuli on four choice keys in each trial. The purpose of this study was to determine whether differences in steady-state matching behavior do exist with different types of stimuli.

\section{METHOD}

\section{Subjects}

The subjects were three adult male White Carneaux pigeons. They were maintained at approximately $80 \%$ of their free-feeding body weights. Water and grit were continuously available in their home cages. Each bird was a subject in a previous experiment involving either stimulus generalization or schedules of reinforcement.

\begin{abstract}
Apparatus
Five BRS/LVE Polacoat pecking keys were mounted at the four corners and center of a $10.5 \times 15 \mathrm{~cm}$ rectangle on a $37.5 \times 37.5 \mathrm{~cm}$ response panel. An in tine stimulus projector (Grason-Stadler \# 156) was mounted behind each key. A peck on any key operated a feedback relay mounted behind the panel. The 6-cm-square magazine opening was located below the keys and was centered between them. Reinforcement consisted of a 4-sec access to Purina pigeon grain during which the magazine was illuminated with a 1.12-W lamp. The experimental chamber was a $43.18 \mathrm{~cm}$ high $\mathrm{x}$ $60.96 \mathrm{~cm}$ wide $\times 41.91 \mathrm{~cm}$ deep plywood box. White noise was present in the experimental room at all times. Programming and recording equipment were located in an adjacent room.
\end{abstract}

\section{Procedure and Results}

Phase 1 of the experiment began as the training phase of another experiment which will not be described here. Due to this, the procedure was more complex than was necessary for this experiment. A trial began when a randomly selected stimulus from the set containing a circle, triangle, " $X$," and square appeared on the center key. When the pigeon pecked this key (an "observing" 


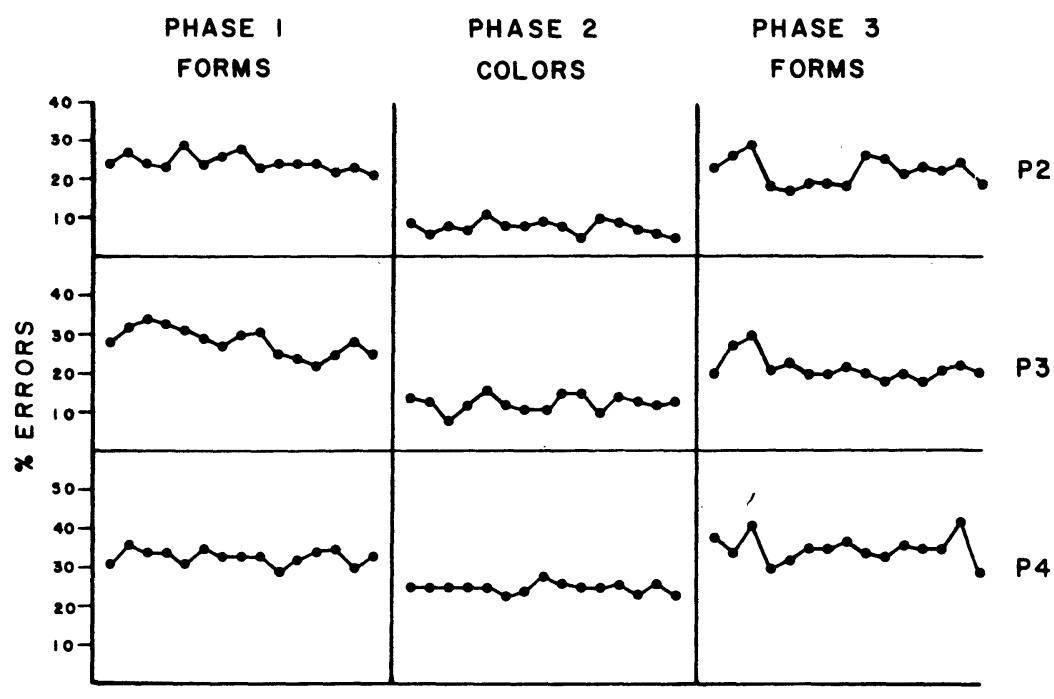

Figure 1. Percentage error levels for the last 15 sessions in each phase of the experi3 ment for all three subjects.

SESSIONS

response), another geometric form from the set appeared. After a series of from three to six forms had appeared, all four stimuli in the set appeared on the four choice keys, and the last stimulus appeared on the center key. The subject was required to peck whichever of the four comparison stimuli matched the final or sample stimulus. A correct match was reinforced with a 4-sec access to mixed grain. An error produced a 15 -sec time-out in which all keys were dark. At the end of the reinforcement presentation or time-out, the next trial began. Each session terminated after $\mathbf{5 0}$ reinforcements.

Stimulus presentations were programmed on punched paper tape in the following manner. Forty-four different sequences of stimuli were arranged in a forward and backward order to make a series of 87 trials. The tape was punched so that each stimulus was correct on approximately one out of every four trials. Four configurations of comparison stimuli could appear on the choice keys so that each stimulus appeared equally often on each key. Each of the four choice keys was also programmed to be correct on approximately one out of four trials. The tape was started at a different point for each subject on each session.

After 50 sessions on this procedure, all subjects had high error levels. and their day-to-day performances were variable. In an effort to improve performance, subjects were gradually shifted to a procedure where four correct matches without an error were required for reinforcement. This was a fixed-ratio (FR4) schedule with an error-contingent reset of the FR. Each correct match was followed by a .3-sec operation of the magazine light, and the fourth correct match in a row was followed by food reinforcement. During the reinforcement presentation, the magazine light was operated and the keys were dark. As a result of the FR4 schedule, both variability and error levels decreased. After 78 sessions, it was determined by inspection that the subjects' matching behavior was stable. The left panel of Figure 1 shows per cent error levels for the last 15 sessions of Phase 1 for all three subjects. Average per cent errors for the last 15 sessions in Phase 1 were 24.6, 28.7, and 33.4 for P2. P3, and P4, respectively. These error levels, using geometric forms as stimuli, were higher than those often found in matching-to-sample experiments.

In Phase 2 of the experiment, the stimuli were changed to colors (red, blue, green, and yellow). Each subject was run on a FR1 for correct matches until it reached either the same per cent error level or the same number of sessions at which it was shifted to FR4 while matching forms. This took 28 sessions for P2, 21 for P3, and 55 sessions for P4. At this point, the procedures in Phases 1 and 2 were identical. Subjects then spent an additional 60-80 sessions on matching colors on a FR4 schedule with an error-contingent reset.
Their error levels had stabilized by the end of these sessions. Figure 1 shows that there was no overlap between the points from Phase 1 and those of Phase 2 for all three subjects. Average per cent errors for the last 15 sessions of color matching were 8.1, 12.9, and 25.3 for P2, P3, and P4, respectively. For all three subjects, performance on color matching was superior to performance on form matching.

In order to show that the superior performance of subjects matching colors over forms was a function of a difference in discriminability of the stimuli rather than the effect of practice, the stimuli were returned to forms in Phase 3 . Error levels immediately increased, although no bird returned to chance performance. Subjects were shifted easily and quickly to a FR4 schedule. At the end of 32 sessions for P2, and 24 sessions for P3 and P4, their performance was stable, and the experiment was terminated. Mean per cent errors for the last $\mathbf{1 5}$ sessions of form matching for P2, P3, and $\mathrm{P} 4$ were 22.4, 22.0, and 35.4, respectively. Figure 1 shows that for all three birds these error levels were higher (with no overlapping points) than those obtained during color matching. For P2 and P4, there was no significant difference $(\mathrm{p}>.05)$ between error levels in Phase 1 and error levels in Phase 3. For P3, per cent errors were only slightly lower in Phase 3 (22.0) than in Phase 1 (28.7), but the difference was statistically significant $(p<.05)$.

\section{DISCUSSION}

These results show that steady-state differences in accuracy, as well as differences in acquisition, do exist between matching with geometric forms and color matching. After extended training, with color stimuli in Phase 2 and form stimuli in Phases 1 and 3, all subjects were substantially more accurate when matching colors. Farthing and Opuda (1974) found that acquisition of color matching was faster than acquisition of form matching, but eventually their subjects, with one exception, were matching both types of stimuli with equal accuracy. The present data indicate that, under some conditions, pigeons are unable after extensive training to match forms as accurately as colors.

The reason for this effect is unclear. It may be the case that colors are more relevant stimuli than shapes to pigeons in their search for food in the natural environment. This would explain their superior accuracy in discriminating colors. To the pigeons, there may have been greater similarity between the form stimuli used in this experiment than between the colors. Hinde (1970) noted that many animals are incapable of discriminating forms which are easily discriminated by humans. On the other hand, it is difficult to know 
if our findings are generalizable to other forms and other colors. For example, if the color stimuli used in this experiment had been very similar in wavelength and equated for brightness, the subjects might have been less accurate in matching colors. Similarly, there may be forms other than those used in this experiment which are less difficult to discriminate.

These data raise a second consideration on the discriminability of stimuli in matching to sample. None of the three subjects learned to match forms with an accuracy greater than $80 \%$ correct. Only one of the three exceeded a $90 \%$ correct criterion when matching colors. This error level appears to represent the final steady state since their matching accuracy was stable after extended training, as can be seen in Figure 1. Previous investigators (Cumming \& Berryman, 1965; Farthing \& Opuda, 1974) have shown steady-state matching accuracy over $90 \%$ correct for both form and color matching. There seems to be an inconsistency between error levels in this experiment and error levels in standard three-key experiments. Several differences exist between their procedures and the procedure of the present study, including the number of stimuli presented before the matching trial, and the total number of stimuli used. A prominent difference is in the number of alternative comparison stimuli used. In the study by Farthing and Opuda (1974), on any trial, subjects were required to choose between two comparison stimuli, and the chance probability of an error was $50 \%$. In this experiment, subjects were required to choose between a set of four comparison stimuli on each trial, so the chance probability of an error was $75 \%$.

A correction for guessing (Magnusson, 1967) which takes account of the differences in the probability of making an error was applied to the mean per cent error levels from this experiment and those from a representative three-key study (Farthing \& Opuda, 1974). The average per cent error level for subjects in the three-key (two comparison stimuli) experiment with color stimuli was $\mathbf{8 \%}$. Corrected for guessing, this becomes $16 \%$. Corrected error levels for the subjects in this five-key (four comparison stimuli) study were $11 \%, 17 \%$, and $33 \%$ for $P 2, P 3$, and $P 4$, respectively.

The average per cent error level for subjects in the three-key experiment with form stimuli was $10 \%$. Corrected for guessing, this becomes $20 \%$. Corrected error levels for the subjects in this five-key experiment were $29 \%, 29 \%$, and $44 \%$, respectively.
These corrected error levels suggest that only one pigeon in this study was substantially less accurate when matching colors than subjects who had only two comparison stimuli. Corrected error levels for form matching show that all subjects in this experiment were less accurate than subjects which matched with two comparison stimuli. It would seem that the number of comparison stimuli may have an effect on the difficulty of form matching but not on color matching. Since a parametric study which varied the number of comparison stimuli was not performed, this interpretation is, of course, speculative. The matter can be clarified only by further research.

\section{REFERENCES}

Blough, D. S. Delayed matching in the pigeon. Journal of the Experimental Analysis of Behavior, 1959, 2, 151-160.

Cumming, W. W., \& Berryman, R. Some data on matching behavior in the pigeon. Journal of the Experimental Analysis of Behavior, 1961, 4, 281-284.

Cumming, W. W., \& BerRyman, R. The complex discriminated operant: Studies of matching to sample and related problems. In D. Mostofsky (Ed.), Stimulus generalization. Stanford: Stanford University Press, 1965. Pp. 284-330.

Farthing, G. W., \& Opuda, M. J. Transfer of matching to sample in pigeons. Journal of the Experimental Analysis of Behavior, 1974, 21, 199-213.

Hinde, R. A. Animal Behavior. New York: McGraw-Hill, 1970.

Magnusson, D. Test theory. Reading, Mass: Addison-Wesley, 1967.

Mello, N. K. Alcohol effects on delayed matching-tosample performance by rhesus monkey. Physiology and Behavior, 1971, 7, 77-101.

(Received for publication February 6, 1976.) 